\title{
La mujer-filósofo o la más "antinatural" de las criaturas. En torno a Simone de Beauvoir y a su obra El segundo sexo
}

María José Guerra Palmero Universidad de La Laguna, España

Resumen

El presente artículo analiza el carácter pionero de una de las obras clave del feminismo, El segundo sexo (1949), contribución fundamental a la filosofía feminista actual y primer marco teórico del feminismo de segunda ola. En este trabajo se analizarán los empleos de la categoría de alteridad para referirla a la existencia de las mujeres. El objetivo principal de la obra de Beauvoir es explicarnos y explicitarnos las condiciones de esa vida femenina expropiada y obligada a permanecer en la mera inmanencia. A partir de esta cuestión emergerá en el feminismo de la segunda ola la conceptualización constructivista de "género". Exploramos, en segundo lugar, la recepción crítica de la obra de Simone de Beauvoir. La falta de reconocimiento, el descrédito y los prejuicios sexistas imposibilitaron hasta casi tres décadas después de su publicación su justa estimación crítica. Finalmente, se presta atención al des/encuentro entre dos generaciones de feministas francesas que representan la propia Simone de Beauvoir, Luce Irigaray y Michelle Le Doeuff.

Palabras clave: Simone de Beauvoir, feminismo, existencialismo, recepción crítica, género. 
Abstract

This article analyzes the pioneering nature of The Second Sex (1949), Simone de Beauvoir's founding text which definitely contributed to contemporary feminist philosophy and provided the first theoretical framework for the Second Wave Feminism. Beauvoir elaborates on "alterity" as the category to refer to women's existence. In this light, this paper first probes into the manifold usages of such category as de Beauvoir explains and renders visible the life conditions of women, who are condemned to live dispossessed and to remain immanent. It is this issue that triggers off within Second Wave feminism the constructivist theorizing of "gender". Secondly, I explore the critical reception of de Beauvoir's work, unacknowledged and prey to sexist discredit and biases for a long time, which eventually retarded its fair assessment three decades afterwards. Finally, the paper attests to the dis/agreement between two generations of French feminists who Simone de Beauvoir herself and Luce Irigaray and Michelle Le Doeuff represent.

Keywords: Simone de Beauvoir, feminism, existencialism, critical reception, gender.

- 1 primer marco teórico del feminismo de la segunda ola fue E proporcionado por la obra de Simone de Beauvoir El segundo sexo, un tratado acerca de la condición de la mujer que impulsó nuevos modos de afrontar analíticamente las estructuras sociales e ideológicas patriarcales en las que las vidas de todos los seres humanos, mujeres y hombres, se desarrollan. Después de analizar los "hechos" - prestando atención a los discursos culturales de la biología, el psicoanálisis y el materialismo histórico-, esta obra desafió los mitos construidos por el imaginario masculino acerca de las mujeres.

El origen de la preocupación de Beauvoir fue activado en el momento en el que se preguntó por su propia identidad. En relación con el esfuerzo por responder a la cuestión de “¿quién soy 
yo?” emergió un continente hundido. La decisión de escribir $E l$ segundo sexo tuvo que ver con el deseo de reflejar su propia identidad a través de una autobiografía. Beauvoir atinó a formular la pregunta justa que abría la caja de sorpresas del patriarcado: “¿Qué ha significado para mí ser una mujer?” (cf. López, 1994; Le Douff, 1985 y Maccall, 1985). La primera respuesta que le dio erró el tiro porque en un primer momento Simone pensó que el hecho de haber nacido y crecido como una mujer no había tenido ningún tipo de consecuencias para su vida. Pensó que la feminidad no había afectado a su identidad real, pero cuando analizó cuidadosamente la cuestión vislumbró una panorámica muy distinta. Al final, llegó a esta contundente conclusión: el mundo en el que vivimos es el mundo de los hombres, hecho por hombres y para hombres, y desde la más tierna infancia yo he sido alimentada con relatos y mitos creados únicamente por los hombres. En este momento se dio cuenta de que había una tarea que realizar: explicar y hacer explícitas las condiciones de posibilidad de tal vida vivida en femenino.

Con la ayuda de las herramientas conceptuales existencialistas $^{1}$ empezó a articular una nueva comprensión filosófica de lo femenino como lo Otro, como alteridad. Las fuentes de su categorización de la alteridad eran hegelianas: la relación entre el hombre y la mujer se asemejaba al asimétrico vínculo entre amo y esclavo. La identidad de la mujer dependía de la identidad del hombre. Ella era definida sólo por referencia al sujeto masculino, pero la diferencia entre esta relación y el episodio de la dialéctica amo y esclavo era que el reconocimiento no era un desenlace posible del choque entre las dos conciencias. El hombre era definido como esencial, la mujer como inesencial; el destino de los hombres es la trascendencia, mientras que las mujeres estaban obligadas a permanecer en la inmanencia. La creatividad y las oportunidades

${ }^{1}$ Sobre el asunto de la identidad en el feminismo y en la teoría crítica, $c f$. M. José Guerra, 1997, pp. 143-154. 
de libertad le estaban prohibidas, tan sólo tenía asignada la tarea repetitiva de la reproducción.

Beauvoir lograba, así, apreciar el núcleo central de la dicotomía: "uno de los términos es afirmado como el único esencial y niega toda relatividad al término que le es correlativo". La mujer es alteridad pura y no puede escapar de ella. Le está vedada la subjetividad, le está denegado el acceso a una conciencia individual. Al no reconocérsela como individuo, la mujer era privada de humanidad plena. Si no podíamos ser individuos libres, si no podíamos ser creadores, si la trascendencia nos estaba prohibida, lo que nos quedaba era el encarcelamiento en el reino de la repetición de la vida natural sin acceso a la cultura humana.

Nuestro destino estaba aherrojado a la relación con los hombres: los roles de esposa y madre eran los "naturales" para nosotras. No podíamos ser pensadas como conciencias separadas que merecen reconocimiento.

Celia Amorós ha señalado que las mujeres estamos forzadas a vivir en el "espacio de las idénticas" (Amorós, 1987), ${ }^{2}$ somos intercambiables porque no hemos, o no nos han dejado, devenir individuo. Nuestro rol es el de mediadoras entre los hombres, tal como Levi-Strauss ha mostrado cuando explica el modo en que los hombres firman y sellan el pacto, la alianza entre ellos. La mujer es un objeto "natural" para ser intercambiado entre hombres, su "importancia" se deriva de este hecho porque ella no puede ser un ser independiente y autónomo. En nuestros días, Donna Haraway ha explicado la verdadera razón de esta identificación: se da por sentado que las mujeres y la naturaleza son objetos susceptibles de ser dominados. La justificación de la dominancia ha sido legitimada en muchos mitos religiosos y culturales: Eva, Pandora, la mujer fatal, o, sin ir más lejos, la ambiciosa ejecutiva con poder y sin compasión. El análisis de los diferentes contextos culturales prueba que la

${ }^{2}$ Cf. de la misma autora "Igualdad e identidad", en Valcárcel (comp.), 1994. 
situación de las mujeres es, básicamente, de desigualdad y opresión. Aunque Beauvoir adopte el marco filosófico existencialista al dar cuenta del sistema patriarcal, rechaza una de las más descollantes ideas de Sartre: que el individuo es responsable de su destino por no ejercer control sobre sus elecciones, una cuestión de mauvaise foi. La ética sartreana de la autenticidad queda desafiada implícitamente porque Beauvoir establece que existen condiciones objetivas de opresión: las mujeres no son culpables de su destino social.

Beauvoir había realizado, en suma, la diagnosis social e ideológica de la opresión de las mujeres. Ahora quedaban, al menos, tres tareas urgentes por llevar a cabo. En primer lugar, el intentar explicar cómo había aparecido y se había desarrollado históricamente la situación desigual de las mujeres. En segundo lugar, el esforzarse en deconstruir los mitos y las tradiciones culturales que devaluaban la posición de las mujeres. Por último, y éste es el asunto en el que estamos interesados ahora, poner de manifiesto la manera en como las mujeres están obligadas a vivir en el marco de la sociedad patriarcal con el resultado de ser expropiadas, hablando existencialmente, de la condición humana como trascendencia. A partir de esta última cuestión emerge la conceptualización del "género". La originalidad de Beauvoir fue reflexionar sobre la identidad de la mujer y darse cuenta de que el género - la dicotomía masculino/femenino- impregna y determina nuestra visión del mundo y los modos en el que vivimos en él. El hecho cultural del género se muestra en esta famosa sentencia: "No se nace mujer, se llega a serlo".

La recepción crítica de $\mathrm{El}$ segundo sexo: prejuicios y hostilidad

Toril Moi ha detectado un curioso fenómeno que afecta a la recepción crítica de la obra de Beauvoir. La presencia de un número desproporcionado de comentarios hostiles, en tono sarcástico y despreciativo (Moi, 1990: 22). Esta autora, una de las representantes 
más importantes de la teoría crítica feminista, se plantea si no está siendo parcial como feminista, y si realmente Beauvoir es una escritora inferior, pero su análisis de la recepción crítica lo que pone de manifiesto es que la estrategia común es "personalizar los asuntos, reducir el libro a la mujer: su objetivo es claramente desacreditar a Beauvoir como hablante, no entrar en un debate con ella" (Moi, 1990: 23). Esta reacción no tiene parangón con el tratamiento crítico de otras importantes escritoras francesas como Simone Weil o Marguerite Yourcenar. Lo que se cuestiona es el derecho de nuestra autora a producir cualquier clase de discurso, a ser sujeto de la enunciación. Defender a Beauvoir es defender, en consecuencia, su derecho a hablar en el contexto de los derechos democráticos a participar en las discusiones públicas ya sean literarias o políticas. Sólo después de restituirle la "autoridad", lo que llama Amelia Valcárcel "la investidura", podremos entrar a valorar sus posiciones. Lo primero es partir de que vamos a tomar a Beauvoir seriamente.

Moi, en este libro del año 90, se siente desalentada porque cuarenta años después del El segundo sexo todavía tengamos que insistir en el derecho a ser tomadas en serio como mujeres y como feministas. Este fenómeno de falta de reconocimiento y de descrédito es analizado por Susan Bordo en The Feminist as Other (1997: 297-312). Ella vuelve a aplicar la categoría de alteridad de Beauvoir en el contexto actual de los estudios feministas. El dictamen generalizado es que ya no se puede "ignorar" o "evitar" las cuestiones relativas al género en la interpretación de la cultura. El presupuesto de esta afirmación es que la perspectiva de género prescribe tan sólo una crítica especializada con implicaciones restringidas para incrementar el caudal de conocimiento acerca de la cultura: "uno se compromete con el género o se implica con la crítica de mayor significado; escoja una opción” (Bordo, 1997: 297).

Esta presuposición puede ser interpretada con las herramientas teóricas propuestas por Beauvoir: el lugar de la alteridad 
contrasta con la esencialidad atribuida a lo universal como seńuelo de lo humano, todo lo que es "definido, reducido y marcado" por su diferencia (sexual, racial, religiosa, etcétera) frente a la norma puede, a lo mejor, ser reconocido, no invisibilizado, pero siempre conlleva el signo de su posición marginal, de su locus periférico. Incluso se puede aplaudir a la feminista, pero siempre como otra. No obstante, Bordo, inspirándose en la misma Beauvoir, mantiene que junto al género otras "condiciones" atribuyen, asimismo, alteridad — pertenecer a minorías raciales, religiosas, culturales, etcétera- El mismo fenómeno detectado a propósito del feminismo puede rastrearse en la discusión acerca de la educación multicultural en la que la diversificación de los currícula no se contempla como un modo de apreciar mayor rigor histórico o de ensayar un enfoque más amplio que el meramente eurocéntrico, sino como satisfacción a las necesidades de determinados grupos. La conclusión, deudora de la de Beauvoir, es que "la alteridad tiene, pues, muchas caras" (Bordo, 1997: 298).

La tesis de Bordo acerca de la recepción crítica de Beauvoir coincide con la de Moi. La primera traducción al inglés de El segundo sexo la hizo un zoólogo no un filósofo/a. Las escasas reseñas estipularon que sólo los hombres hacían filosofía, y que las mujeres, todo lo más, podían escribir con tino acerca de su propia condición. Otro comentario estipuló "2 3/4 libras", señalando con ello el peso del libro, su materialidad inmanente, como si fuera el peso de un bebé, haciendo visible la reducción de la escritura de una mujer al acto de dar a luz. Bordo concluye: "Así Beauvoir, la más antinatural de las criaturas, una mujer filósofa, fue puesta en el lugar que le correspondía”.

Conjugando las estimaciones de Moi y Bordo, lo que nos queda es que sólo en la década de los noventa empezamos a encontrar una justa y ajustada recepción crítica que se merece $E l$ segundo sexo. Es tiempo de redimir la deuda con Beauvoir. 
Volviendo a Moi, con lo que nos encontramos es con el hecho que de 1950 a 1980 el cliché sexista fue el determinante. Su tesis es que la crítica había reducido "el libro a la mujer" (Moi, 1990: 27), y que se utiliza lo personal para desacreditar las opciones políticas de Beauvoir (Moi, 1990: 33 y ss.). Ciertos motivos comunes son constantes, por ejemplo, alusiones a su apariencia, carácter, vida privada o moralidad. Ello implica esta recurrencia: "lo que diga, escriba o piense una mujer es menos importante y menos interesante que lo que ella es". La asignación de superficialidad y frivolidad es otra constante y, además, la denegación de su capacidad como escritora por acudir a lo autobiográfico. ¿Qué tendríamos que decir, entonces, de las confesiones de Agustín, los ensayos de Montaigne o lo correspondiente en Rousseau? Por otra parte, los mismos críticos veneran, por ejemplo, a Cannetti por su hacer autobiográfico, mientras denigran a Beauvoir (Moi, 1990: 32.). Los escritos políticos, filosóficos o sobre asuntos no personales se invisibilizan.

La inclemente crítica la acusa, por otra parte, de que todos sus personajes femeninos son Beauvoir. Se le atribuye egoísmo, narcisismo e ingenuidad. Simone de Beauvoir, al ser identificada como escritora con todos sus personajes, pasa a ser todas las mujeres. La misma autora se reía de esta simplificación que le negaba autoría e imaginación literaria. Sólo está interesada en ella misma y la autosatisfacción es desagradable, por otra parte, en una mujer. Además, y ésta es la puntilla final: "Se lo debe todo a un hombre", es decir, a Sartre. Sus escritos son efectos de esta relación. Su trabajo más que de creación es de "popularización", un trabajo "menor", un epígono.

Pero a todo lo anterior se le suma la que podemos llamar la "interpretación patologizadora". Beauvoir es una mujer con problemas de personalidad. Una mujer no femenina, no maternal. Esto alimenta la crítica política de que sus opciones son ajenas a la 
preocupación por el bienestar de la humanidad, porque no muestra compasión. A nadie, por supuesto, se le ocurre descalificar así, en estos términos, a un político. En resumen, se la acusa de "falta de humanidad", y si escribe de política es porque desplaza a ese ámbito sus problemas personales. Son efectos de la histerización del cuerpo de la mujer: que la emulan al prototipo de la bruja, de la arpía. Moi apunta un paralelismo entre la crítica a Beauvoir y a Woolf: ambas pagan caro no encarnar al ángel del hogar (Moi, 1990: 34).

Otro foco de descalificación se asimila al estereotipo de la marisabidilla. Se atacan las pretensiones intelectuales de Beauvoir (Moi, 1990: 51). Su ironía, si no tiene la complicidad del lector, se desvanece. Es una falsa intelectual. El derecho a dedicarse a la filosofía por parte de una mujer ha sido duramente contestado.

Pero esto no es todo. Para explicar la situación francesa tenemos que aludir al hecho de que las modas intelectuales en los años sesenta y setenta consagrarán la reacción antiexistencialista de la mano del protagonismo filosófico del estructuralismo, del postestructuralismo, del psicoanálisis a lo Lacan y, posteriormente, del mismo postmodernismo en los años ochenta. Esto en lo que se refiere al contexto filosófico global. Pero, en consonancia con el poder de influencia de este contexto, el feminismo francés y sus desarrollos más conocidos tomaron como punto de partida una suerte de matricidio teórico (cf. Moi, 1988: 101-112). Si Beauvoir se había planteado si había que quemar a Sade, sus "hijas" o "hermanas menores" optaron por quemar El segundo sexo. La manzana de la discordia fue la recepción feminista del psicoanálisis lacaniano.

Beauvoir e Irigaray o de la incomprensión de las "hermanas"

Para el feminismo de la diferencia francés, Beauvoir había errado el camino al querer la igualdad para la "mujer independiente" capaz 
de abandonar la inmanencia y atarse a la trascendencia, porque esta "igualdad" no era otra cosa que una homologación acrítica con los varones. En un feminismo dominado por el psicoanálisis lacaniano matar a la madre parecía casi una exigencia incontrovertible.

La misma Luce Irigaray nos cuenta el otro lado de esta historia de incomprensiones en el feminismo francés (Irigaray, 1992: 7-11). Primero, concede la importancia crucial de Beauvoir al poner de nuevo, tras un largo paréntesis (el que separa la primera ola de la segunda ola), la atención sobre el hecho de la explotación de las mujeres. Segundo, alude al papel de "modelo", o quizás podríamos decir de contramodelo, que al relatar su vida y al entretejerla con su análisis filosófico proporcionó nuevos horizontes vitales, públicos - escritora, filósofa, profesora, activista...- y privados -miembro de una pareja "flexible" con una sexualidad no convencional-, para la aspiración de las mujeres a la libertad. Finalmente, su soporte público del emergente feminismo de los ańos setenta como intelectual de reconocido prestigio fue una inestimable ayuda para el movimiento.

Pero llegó la "época del psicoanálisis”, y con ella la caída en desgracia de Beauvoir, cuando la nueva generación de feministas empezó a ver las cosas de otra manera. El incidente que envolvió la primera obra de Irigaray, Speculum de la otra mujer, tuvo la callada por respuesta de parte de Beauvoir. Al envío de la obra no respondió nunca, e Irigaray se vio no sólo privada del apoyo de la "hermana mayor", sino expulsada de la ortodoxia lacaniana y expropiada de su posición universitaria. La recepción crítica de Speculum libro en el que se sienta en el diván psicoanalítico a la gran tradición filosófica occidental - también merecería un estudio aparte, porque en esta obra se vuelve a repetir la virulencia hostil, aunque también la admiración sin tacha de las tesis de la autora. Todo esto nos devuelve a la misma pregunta: ¿por qué tanta irritación ante las obras rupturistas de las mujeres filósofas-feministas? El silencio 
de Beauvoir, a título de conjetura, se puede explicar al menos refiriéndolo a dos factores: por un lado, su animadversión frente al psicoanálisis expuesta claramente en $E l$ segundo sexo; por otro, su desagrado ante la condenación plena que Irigaray hacía de toda la tradición filosófica de la que, sin embargo, Beauvoir era heredera, aunque albergará hacia ella una tensa ambivalencia. Pero, quizás, el reactivo esencial fue la propuesta de la joven Irigaray de un feminismo de la diferencia que podía ser interpretado como una vuelta sin retorno al Eterno Femenino. Beauvoir no podía renunciar a la pretensión a la trascendencia, no podía quedar presa de la inmanencia inscrita en el cuerpo de las mujeres al servicio del mantenimiento de la vida. Beauvoir no quería retornar a la caverna platónica, metáfora del útero materno. Aquí acaban nuestras pobres conjeturas acerca del silencio de Beauvoir.

Para Irigaray existe todo un continente por descubrir tras el limitado horizonte de la igualdad entre los sexos. Su objetivo es que las mujeres debemos acceder a una nueva identidad, y que para ello hay no sólo que deconstruir la tradición cultural heredada que desprecia y desvaloriza a las mujeres, sino renovar el orden simbólico, de manera que las mujeres puedan sentirse orgullosas de serlo. La tesis de esta autora es que a la filosofía le queda por pensar lo impensado: la diferencia sexual. El gran tema sepultado por el hilo androcéntrico de la filosofía occidental: "Son siglos de valores socio-culturales los que hay que revisar y transformar, empezando por las mujeres mismas" (Irigaray, 1992: 9).

La neutralización del sexo no es posible. El género no puede dejar detrás de sí el sexo como un impensado. Potenciar simbólicamente la sexualidad femenina al margen de los modelos falocráticos es el objetivo. Frente a la emancipación de la naturaleza, que plantea Beauvoir, Irigaray se empeña en seguir pensando la mediación entre la vida y la cultura, entre lo natural y lo cultural que ha sido encarnada por las mujeres. El recuerdo de Irigaray para con 
Beauvoir muestra su reconocimiento, pero también la amargura del desencuentro entre dos generaciones de feministas francesas que desencadenó la disputa en el seno del feminismo entre partidarias unilaterales de ambos enfoques.

\section{Otro diagnóstico: la "genialidad de la inadecuación"}

En 1989, Michele Le Doeuff presentó El estudio y la rueca. De las mujeres, de la filosofía, etc. (1993), donde trata a Beauvoir en el contexto más general de las difíciles relaciones entre las mujeres y la filosofía, dada la falta de "naturalidad" de la mujer filósofa, y dado que si a ésta ya de por sí dudosa condición se le anudaba la de feminista, denominación que Beauvoir abraza tardíamente en los años setenta, clarifica las constantes sexistas y antifeministas de la hostil recepción crítica de su obra.

Ya Moi nos advertía que el ensayo de Le Doeuff representa un turning point en la recepción de El segundo sexo. Esta autora valora la actualidad de las descripciones de los hechos de la opresión de las mujeres en la obra de Beauvoir, porque siguen siendo enormemente vívidas, y plantea que el instrumental teórico, la moral existencialista, es el que ha perdido fuelle. No obstante, Beauvoir, al describir la opresión, habría desafiado calladamente la figura sartreana de la "mala fe", llevando al existencialismo, que para esta autora "no es un feminismo", más allá de sus límites. ¿Cómo entra Beauvoir a formar parte de la comunidad de los filósofos? En el dudoso papel de "admiradora-enamorada", dominada por el "complejo de Heloísa” (Le Doeuff, 1993: 93), nuestra autora se deja atrapar por la subsidiariedad. El estereotipo de las uniones filosóficas y el que el libro en cuestión fuera ofrecido por la misma Beauvoir como "confirmación singular de la validez del sartrismo" —_[...] tu verdad me hará libre” (Le Doeuff, 1993: 93) — abren la puerta al daño que los maridajes teóricos unilaterales han propicia- 
do al feminismo. El feminismo francés, especialmente, ha vivido conmovido por la presión a definirse en función de los grandes filósofos: Lacan, Foucault, Derrida (Jardine, 1986), etcétera. La causa de las mujeres se enarbola como motivo de "gloria de un maestro contra sus rivales" (Le Doeuff, 1993: 94). Con esta necesidad de vinculación monogámica se acaba con lo que es la verdadera esencia del feminismo: la voluntad de independencia intelectual a la que debemos sumar el ejercicio sistemático de la hermenéutica de la sospecha frente a las obras de los "padres" y/o "hermanos mayores". Este hecho, del que en la actualidad nos distanciamos, ha tenido que ver con lo que ya ponía de manifiesto Moi: las mujeres no estamos autorizadas a hablar y menos a argumentar filosóficamente. El refugiarse en una corriente o hacerse deudora de un maestro permite acceder a una deriva limitada de autorización. Beauvoir no podía disponer de una genealogía femenina y feminista como la que disponemos hoy cincuenta años después de $E l s e-$ gundo sexo. Tenía que hablar como si fuese la primera, y este hecho la situaba en el vacío de la incomprensión. No obstante, Le Doueff demuestra que la tutela teórica de Sartre funcionó como ficción y, quizás, como autoengaño. En los textos sartreanos, las mujeres no ostentan el estatus de sujetos, son reducidas a sexualidad, y recriminadas por rechazar el deseo masculino, en esto consiste su mala fe. La temática existencialista se "transformó" para poder ser de alguna utilidad al análisis feminista. Beauvoir no quiere al modo cartesiano-sartreano construir un sistema, su punto de partida son los valores morales de la autenticidad y la libertad, y ellos dirigen el trabajo descriptivo de la opresión. De esta manera, se producen silenciosos desplazamientos. La "mala fe" sartreana sirve al objetivo de sondear la conciencia del otro. Beauvoir se contenta, en cambio, con detectar contradicciones en los discursos de los otros - ya sean míticos, filosóficos o literarios-, y asigna la culpa a "un conjunto de tradiciones nefastas y de ideologías perversas" (Le Doeuff, 
1993: 142). El caso es que el famoso concepto de "mala fe" es desautorizado por la misma Beauvoir, quien remite los autoengaños a la situación que impide la autotransparencia de los sujetos. El efecto de la discriminación es profundo y lo impregna todo, Beauvoir es enormemente benévola en sus juicios, a decir de Le Doueff. El caso es que la desaparición del imaginario masculino, sobre todo la idea de superioridad, hace que la "mala fe" se eclipse en la obra de Beauvoir. ¿Hacia dónde se traslada el énfasis? La exterioridad debe proporcionar "medios concretos" para afirmarse como sujeto, no vale simplemente mi vocación de trascenderla. El problema de la mujer es que está privada del mundo. Todo se alía para destinarla a la impotencia. Le Doeuff ironiza: “¿Pueden ser las guarderías una preocupación del ego absoluto?” (1993: 151).

De hecho, el enigma reza así, ¿cómo podía en 1949 Simone de Beauvoir escribir El segundo sexo dado el aislamiento al que estaban sometidas las mujeres y a la ausencia de conciencia de la misma situación de opresión? La diferencia con el momento presente es abrumadora, toda vez que hemos rescatado parte de la historia que nos permite hablar de nosotras las mujeres, y que hemos articulado el logro de la autonomía de las mujeres de manera colectiva, ya que sabemos que o bien nos "liberamos" todas o no se libera ninguna, con lo que la idea de solidaridad feminista es la que anima la pugna por acrecentar las mismas cotas de autonomía.

El dogma del que se ha alimentado el pensamiento francés es de la dicotomía de lo Mismo y lo Otro. La "alteridad", este concepto que tanto juego da a Beauvoir, casi nos priva de El segundo sexo si se toma como traducción la posibilidad de una polaridad ontológica que es a lo que Levinás reduce la misma feminidad. No obstante, el incluir en la relación entre los términos la reciprocidad salva a nuestra autora de abandonar el proyecto. La pretensión de reconocimiento que eleva una conciencia es recíproca. Si a esto se suma la negativa de Beauvoir a esencializar o a naturalizar la condición femenina, nos encontramos con la denuncia de la "ar- 
bitrariedad cultural" que exige el sometimiento de las mujeres privándolas de mundo $y$, así, privándolas del acceso a la subjetividad. La exigencia de libertad es tan sólo "una simple afirmación de la vida", porque la opresión de las mujeres las condena a muerte en vida. La "mala fe" sartreana se ha disuelto por el camino. La conclusión de Le Doeuff es la siguiente: "Cualquier filosofía, aplicada a un campo conflictivo por alguien que está comprometido en ese campo y que plantea en relación a él objetivos prácticos, sufre modificaciones apreciables" (1993: 179).

El siglo XX se recordará, cuando pasen los años, como el siglo en el que la pauta patriarcal se quiebra, aunque es verdad que parcialmente y alumbrando multitud de conflictos. El segundo sexo quedará, sin duda, como uno de los libros más influyentes del siglo. Ha sellado, en suma, la conjunción fructífera entre filosofía y feminismo en pos de la libertad y la igualdad entre todos los seres humanos.

\section{Bibliografía}

Amorós, Celia, 1987, "Espacio de los iguales, espacio de las idénticas", en Arbor, núm. 503-4, noviembre-diciembre, s.e., s.l.e., pp. 113-27.

Beauvoir, S., 1998, El segundo sexo, Cátedra, Madrid.

Bordo, Susan, 1997, “The Feminist as Other", en Philosophy in a Feminist Voice (ed. de Kourany, Janet), Princeton University Press, New Jersey, pp. 297-312.

Guerra, María José, 2001, Teoría feminista contemporánea. Una aproximación desde la ética, Editorial Complutense, Madrid.

Guerra, María José, 1997, “¿'Subvertir’ o ‘situar' la identidad? Sopesando las estrategias feministas de Judith Butler y Seyla Benhabib”, en Daimon. Revista de Filosofia, núm. 14. s.e., s.l.e. 
Irigaray, Luce, 1992, “A manera de aviso. Iguales o diferentes”, en Yo, tú, nosotras, Cátedra, Madrid, pp. 7-11.

Jardine, Alice, 1986, Gynesis, PUF, París, 281 pp.

Le Doeuff, Michele, 1993, El estudio y la rueca. De las mujeres, de la filosofía, etc., Cátedra, Madrid, 473 pp.

_, 1985 , "Simone de Beauvoir y el existencialismo", en Desde el feminismo, núm. 0, diciembre.

López Pardina, M. Teresa, 2006, "Simone de Beauvoir", en Veinte pensadoras del siglo XX (eds. de María José Guerra y Ana Hardisson), Nobel, Oviedo.

__ 1994, "El feminismo de Simone de Beauvoir", en Historia de la teoría feminista (coord. por C. Amorós), Instituto de Investigaciones Feministas, Madrid.

Maccall, D., 1985, "Existencialismo o feminismo", en Desde el feminismo, núm. 0 , diciembre, s.e., s.l.e.

Moi, Toril, 1990, "Politics and the Intellectual Woman: Clichés in the Reception of Simone de Beauvoir's Work", en Feminist Theory \& Simone de Beauvoir, Blackwell, London, pp. 21-60. 